\title{
Audit of results of operations for infantile pyloric stenosis in a district general hospital
}

\author{
C A Eriksen, C J Anders
}

\begin{abstract}
Because of the proposal that infants with hypertrophic pyloric stenosis should only be treated by surgeons with an interest in paediatric surgery, we carried out a retrospective study to audit our experience in a district general hospital. Forty six infants over a five year period underwent pyloromyotomy. There were no deaths, and 36 infants (78\%) made uneventful recoveries. Perforation of the duodenal mucosa occurred during the operation in 11 patients, and eight complications developed in six of these infants. There were seven wound infections, and two patients had vomiting that lasted four days or longer after their operations. There were no long term feeding problems.

The results of this study show that such patients can be successfully treated in district general hospitals, and three areas merit special attention: meticulous surgical technique, the use of prophylactic antibiotics, and early graduated feeding.
\end{abstract}

In a recent report from a regional centre in London, it was suggested that infants being treated in a district general hospital for hypertrophic pyloric stenosis should instead be treated by surgeons with a special interest in paediatric surgery. ${ }^{1}$ An earlier study from a district general hospital in England was cited, and the proposal was made because of the high complication rate. ${ }^{2}$ These prompted the present retrospective study: our aims were, firstly, to audit our own experience at a district general hospital in treating infants with pyloric stenosis, and, secondly, to propose any necessary changes in management so that such patients may be successfully treated by general surgeons in district general hospitals.

\section{Patients and methods}

During the five year period July 1984 to June 1989, 46 infants with hypertrophic pyloric stenosis were referred for treatment to the department of surgery at this hospital. There were 43 boys (93\%) and three girls. The mean age at presentation to hospital was 5 weeks (range 2-14). Although most of the babies had had symptoms of postprandial non-bilious vomiting for a mean duration of six days, four infants (9\%) had been ill for three weeks or longer. A family history of pyloric stenosis was present in three cases (mother, maternal aunt and uncle, and paternal uncle), and 24 (52\%) were first born children. Four babies were born prematurely, and associated abnormalities were encountered in two: one child had a duplex ureter, and a second had Down's syndrome.

All 46 infants were admitted to the special care baby unit under the care of a paediatrician. On initial examination, 14 infants $(30 \%)$ were clinically dehydrated (mild, $n=6$ and moderate, $n=8$ ), and they were duly treated with appropriate intravenous fluid replacement. Infants with electrolyte imbalances were also treated. The diagnosis of infantile hypertrophic pyloric stenosis was confirmed clinically in 44 of the 46 infants after a test feed had been given and the pyloric 'tumour' had been palpated. The remaining two infants were diagnosed on clinical ground alone. Plain abdominal radiographs showed a distended stomach $(n=2)$, and barium studies $(n=5)$ were suggestive of gastric outlet obstruction. An ultrasonographic examination was performed in one case before the barium study. After re-examination by the surgical team on call nasogastric tubes were passed in each infant, and the stomach contents aspirated. Operative treatment was planned within the following 24 hours.

All infants underwent operation under general anaesthesia with endotracheal intubation. The operations were carried out by consultant surgeons $(n=13)$, senior surgical registrars $(n=$ $19)$, and post-fellowship registrars $(n=14)$. One preoperative dose of flucloxacillin was given in 32 cases $(70 \%)$.

In each case a transverse skin incision was made in the right upper quadrant of the abdomen and the diagnosis was confirmed. A longitudinal pyloromyotomy was made with a scalpel with a number 15 blade, and the deepest muscle fibres adjacent to the mucosa were split with a pair of mosquito artery forceps. This procedure was done to minimise the risk of damage to the duodenal mucosa. The integrity of the exposed mucosa was tested in all cases by instilling air through the nasogastric tube. The abdominal wall was closed in layers in 19 infants, and mass closure was used for the remaining $27(59 \%)$. Various suture material were used for closure: polyglactin $(n=19)$, polyglycolic acid $(n=12)$, chromic catgut $(n=10)$, polydioxanone $(n=4)$, and polyamide $(n=1)$. The skin was closed with a subcuticular suture of the same material, except in three infants in whom the skin edges were approximated by an adhesive polyvinyl membrane (Opsite, Smith and Nephew).

The postoperative oral feeding regimen was supervised by the paediatricians and nursing staff in the special care baby unit (table). Feeding started four hours after operation with small sips of Dioralyte (Rorer) and the amount was
Correspondence to: Mr Eriksen.

Accepted 10 May 1990 
Oral feeding regimen for infants who have had a Ramstedt's pyloromyotomy for hypertrophic pyloric stenosis

\begin{tabular}{lll}
\hline Feeding rate & Feed & Amount $(\mathrm{ml})$ \\
\hline First 4 hours after operation no feed given then: \\
Every hour & Dioralyte* $^{*}$ & 4 \\
& Dioralyte* $^{*}$ & 4 \\
& Dioralyte $^{*}$ & 8 \\
Every 2 hours & Dioralyte* & 12 \\
& Quarter strength milk & 15 \\
& Quarter strength milk & 20 \\
Quarter strength milk & 30 \\
Every 3 hours & Half strength milk & 30 \\
& Half strength milk & 45 \\
& Half strength milk & 60 \\
& Half strength milk & 60 \\
Every 4 hours & Three quarter strength milk & 75 \\
& Three quarter strength milk & 90 \\
& Full strength milk & 90
\end{tabular}

*Sodium $35 \mathrm{mmol} / \mathrm{l}$, potassium $20 \mathrm{mmol} / \mathrm{l}$, chloride $37 \mathrm{mmol} / \mathrm{l}$ bicarbonate $18 \mathrm{mmol} / 1$, and glucose $200 \mathrm{ml} / \mathrm{l}$.

gradually increased hourly. If the baby vomited, the subsequent amount of feed was not increased. After discharge from hospital each child was followed up at the paediatric and surgical clinics.

\section{Results}

The clinical diagnosis of hypertrophic pyloric stenosis was confirmed at operation in all 46 patients, including the two infants in whom the diagnosis had been made on clinical grounds alone. After Ramstedt's pyloromyotomy, 36 infants $(78 \%)$ made uneventful recoveries, and in 44 infants (96\%) vomiting ceased within three days of operation. No child required a repeat pyloromyotomy, and there were no deaths. The median postoperative hospital stay was four days (range two to 12).

The duodenal mucosa was breached during the operation in 11 infants (24\%): consultant $(n=1)$, senior registrar $(n=5)$, and registrar $(n=$ 5 ), and in each instance this was repaired with a fine chromic catgut suture. Six of these infants had postoperative complications including vomiting ( $n=3$, in whom it was mild and short lived in two), wound dehiscence $(n=2)$, incisional hernia $(n=1)$, wound infection $(n=1)$, and gaping wound edges after skin closure by Opsite dressing $(n=1)$. Nine of these 11 infants had received preoperative antibiotic prophylaxis, and the mean postoperative hospital stay was significantly longer in this subgroup compared with the remaining patients $(7 \cdot 1 \mathrm{com}$ pared with 5.0 days, $p<0.02$, Mann-Whitney $U$ test).

Superficial wound infection occurred in seven infants (15\%), and Staphylococcus aureus was isolated from the wounds in two cases. All infections were mild and none required surgical drainage. In three cases, one dose of antibiotic had been given preoperatively. There were two wound dehiscences, both of which were associated with breached duodenal mucosa. In each case, the wound burst on the second postoperative day, and both infants had had mass closure of the abdominal wall with polyglycolic acid sutures. There was one incisional hernia, which occurred after 25 days; the abdominal wall had been closed in layers with chromic catgut sutures. In three infants, the wound edges gaped and had to be taped with Steri-strips; in two of these children Opsite had been used for skin closure.

Thirteen infants had vomited postoperatively $(28 \%)$. This was mild and short lived (lasting two or three days) in 11 cases, and lasted four days or longer in two children. In all infants, however, the vomiting settled spontaneously before discharge from hospital. Other complications encountered were urinary tract infection $(\mathbf{n}=1)$ and eye infection with $S$ aureus $(\mathbf{n}=1)$.

The median follow up period was six weeks, and seven children were followed up for longer than two years. There were no long term feeding problems, although two children had mild gastro-oesophageal reflux. Only two infants were slow to gain weight during the follow up period.

\section{Discussion}

The number of infants with pyloric stenosis treated each year at this hospital is similar to those from other district general hospitals in England. ${ }^{2}{ }^{3}$ Our incidence compares favourably with the reported incidence of the condition in the Western world of between 1 and 3/1000 live births. ${ }^{45}$ The number of such patients treated in the layer regional centres is greater, partly as a result of referrals from peripheral hospitals. ${ }^{1}$

The diagnosis is made clinically by the palpation of the hypertrophied pylorus, after a small 'test feed' has been given to the infant. This is facilitated by aspiration of the stomach contents through a nasogastric tube, which is then removed. ${ }^{6}$ In the present study, the pyloric 'tumour' was palpated in 44 of the 46 infants (96\%), a rate similar to other reports. ${ }^{126}$ The hypertrophied pylorus can be somewhat elusive, however, and multiple examinations are commonly required to locate it. Radiological or ultrasonographic investigations, or both, have been suggested to improve the diagnostic accuracy, but are not routinely performed in these patients. Indeed, the request rate for such examinations in the United Kingdom varies between 6 and $23 \% .^{1-3}$ This differs from the clinical practice in the United States; it was recently reported that roughly $80 \%$ of such infants were referred for one or other diagnostic imaging procedure. ${ }^{6}$ The authors concluded that such investigations are unnecessary if the diagnosis can be made clinically. Even in difficult cases where there is clinical doubt, however, barium studies are often equivocal. ${ }^{7}$ Ultrasongraphic examination, first performed in 1977 in such patients, ${ }^{8}$ is complicated by subjective interpretation, and differing diagnostic criteria have been reported. ${ }^{9} 10$ Its use for diagnosis seems doubtful. ${ }^{11}$

In the present study, the consultant surgeons, senior registrars, and post-fellowship registrars each carried out similar numbers of operations, but the postoperative complications were slightly more common among patients operated on by the registrars. The duodenal mucosa was performed on 11 occasions, and was associated with eight postoperative complications. Of these eight, however, five were mild (vomiting, wound infection, and gaping wound edges) and the remaining three (wound dehiscence and 
incisional hernia) were probably related more to the surgical technique than the perforation of the duodenal mucosa. Indeed, in a recent retrospective study, surgical technique was the only variable significantly associated with subsequent wound infection. ${ }^{12}$ It is vitally important to recognise a perforation in the duodenal mucosa and to repair it immediately. Mosquito artery forceps were used to prise apart the deepest muscle fibres of the pyloric, but recently the rounded end of the scalpel holder has been reported to be superior in protecting against mucosal perforation. ${ }^{1}$

Almost half of the postoperative complications in the present study were related to the wound. Meticulous attention to the technique of wound closure is essential in order to reduce this incidence. A variety of suture materials was used for abdominal wall closure, although chromic catgut was used only in the early part of the study. Recently, a move has been made towards using polydioxanone rather than the other materials. This suture, when used in a mass closure technique, seems to be effective in preventing wound dehiscence and is associated with a significantly lower wound infection rate. ${ }^{12}$ Wound dehiscence is more common after operations done under local anaesthetic,,$^{2}$ and this is related to the technical difficulty of closing a wound without adequate muscle relaxation. ${ }^{1}$

The wound infection rate is the present study is comparable with quoted rates of $5-17 \%$ from other centres. ${ }^{1-3} 512$ The umbilicus is an important source of $S$ aureus infection, and has been implicated in the aetiology of wound infection. ${ }^{13} 14$ A preoperative dose of an antistaphylococcal antibiotic was given in almost $70 \%$ of our patients and at operation the umbilicus was cleaned with chlorhexidine. Other authors advocate, in addition, sealing off the umbilicus with adhesive tape, but the effect of this in reducing the wound infection rate is limited. ${ }^{113}$

Vomiting during the first two postoperative days is common and self limiting. ${ }^{1-3}$ It is thought most likely to be the result of inhibition of gastric peristalsis during the first 24 hours after operation, with or without associated gastro-oesophageal reflux. ${ }^{13}$ Preoperative oesophagitis and the use of general anaesthesia have also been implicated. ${ }^{12}$ To circumvent this early vomiting, a delayed feeding regimen was proposed whereby the infants were fasted for the first 24 hours after the operation. ${ }^{14}$ As little importance is placed on this early vomiting these patients may be fed orally within the first four hours of operation, and tolerate it well provided that the feeds are introduced slowly.

No patients in the study had any long term feeding difficulties. After a complete pyloromyotomy this is normal, as the hypertrophy at the pyloric regresses at a variable rate and the pylorus achieves a normal size at about 12 weeks after operation. ${ }^{8}$ Mild gastro-oesophageal reflux was evident in two children, however, but this is a recognised problem in a small proportion of patients after Ramstedt's pyloromyotomy.

The results of this study show that infants with hypertrophic pyloric stenosis can be successfully treated by general surgeons in a dis- trict general hospital. In order to improve our results and more closely match those of regional centres, however, there are three areas that merit special attention: meticulous attention to surgical technique, the use of preoperative prophylactic antibiotics, and gentle and graduated introduction of oral feeding after operation.

1 Zeidan B, Wyatt J, Mackersie A, Brereton RJ. Recent results of treatment of infantile hypertrophic stenosis. Arch Dis Child 1988;63:1060-4.

2 Bristol JB, Bolton RA. The results of Ramstedt's operation in a district general hospital. Br F Surg 1981;68:590-2.

3 Gray DW, Gear MW, Stevens DW. The results of Ramstedt's operation: room for complacency? Ann R Coll Surg Engl 1984;66:280-2.

4 Habbick BF, To T. Incidence of infantile hypertrophic pyloric stenosis in Saskatchewan, 1970-1985. Can Med pyssoc $\mathcal{F} 1989 ; 140: 395-8$.

5 Tan KC, Bianchi A. Circumferential umbilical incision for pyloromyotomy. Br $\mathcal{F}$ Surg 1986;73:399.

6 Breaux CW, Georgeson KE, Royal SA, Curnow AJ. Changing patterns in the diagnosis of hypertrophic pyloric stenosis Pediatrics 1988;81:213-7.

7 Larsen GL. Limitations of roentgenographic examination in the diagnosis of infantile hypertrophic pyloric stenosis. Surgery 1966;60:768-72.

8 Teele RL, Smith EH. Ultrasound in the diagnosis of idiopathic hypertrophic pyloric stenosis. $N$ Engl f $\mathrm{Med}$ 1977;296:1149-50.

9 Tunell WP, Wilson DA. Pyloric stenosis: diagnosis by realtime sonography, the pyloric muscle length method. $f$ Pediatr Surg 1984;19:795-9.

10 Khampaparad T, Athey PA. Ultrasound diagnosis of hypertrophic pyloric stenosis. I Pediatr 1983;102:23-6.

11 Carver RA, Okorie NM, Steiner GM, Dickson JAS. Infantile hypertrophic pyloric stenosis-diagnosis from the pyloric muscle index. Clin Radiol 1987;138:625-7.

12 Rao N, Youngson GG. Wound sepsis following Ramstedt pyloromyotomy. $B r$ f Surg 1989;76:1144-6.

13 Doig CM, Wilkinson AW. Wound infection in a children's hospital. Br f Surg 1976;63:647-50.

14 Leaky A, Fitzgerald RJ. The influence of delayed feeding on postoperative vomiting in hypertrophic pyloric stenosis. $B r$ F Surg 1982;69:658-9.

\section{Commentary}

An article in this journal in 1988 by Zeidan et al reviewed the management of 106 babies with hypertrophic pyloric stenosis. ${ }^{1}$ Their patients were admitted under the care of one surgeon working in two children's hospitals: Queen Elizabeth, Hackney, and the Hospital for Sick Children, Great Ormond Street. For those who may have forgotten, the final sentence in the article suggested that, ' . . . healthy infants being treated in district general hospitals should be treated by surgeons and anaesthetists with a special interest in paediatric surgery'. This sentiment prompted the present authors to review experience in their hospital. The preoperative and postoperative management was undertaken by the paediatricians and the surgery was performed by the 'on call' surgical team. There were no operative deaths in either series. However, morbidity rates differed considerably: dehiscence was $0 \%$ compared with $4 \%$ present series, wound infection $5 \%$ compared with $15 \%$, and duodenal perforation $8 \%$ compared with $24 \%$. Both series showed a high degree of accuracy of clinical diagnosis and in neither series was postoperative stay prolonged. On the basis of these results, the authors conclude that pyloric stenosis can be successfully managed by general surgeons and by inference by surgeons without a particular interest in the surgery of childhood. They do not discuss whether or not they consider this optimal management. 